\title{
Approximation Algorithm for k-node Connected Subgraphs via Critical Graphs
}

\author{
[Extended Abstract] \\ G. Kortsarz \\ Rutgers University \\ 3rd. and Penn Streets \\ Camden, USA \\ guyk@crab.rutgers.edu \\ Z. Nutov \\ The Open University \\ Klasner 16 \\ Ramat Aviv, Tel Aviv, Israel \\ nutov@shaked.openu.ac.il
}

\begin{abstract}
We present two new approximation algorithms for the problem of finding a $k$-node connected spanning subgraph (directed or undirected) of minimum cost. The best known approximation guarantees for this problem were $O\left(\min \left\{k, \frac{n}{\sqrt{n-k}}\right\}\right)$ for both directed and undirected graphs, and $O(\ln k)$ for undirected graphs with $n \geq 6 k^{2}$, where $n$ is the number of nodes in the input graph. Our first algorithm has approximation ratio $O\left(\frac{k}{n-k} \ln ^{2} k\right)$, which is $O\left(\ln ^{2} k\right)$ except for very large values of $k$, namely, $k=n-o(n)$. This algorithm is based on a new result on $\ell$-connected $p$-critical graphs, which is of independent interest in the context of graph theory. Our second algorithm uses the primal-dual method and has approximation ratio $O(\sqrt{n} \ln k)$ for all values of $n, k$. Combining these two gives an algorithm with approximation ratio $O\left(\ln k \cdot \min \left\{\sqrt{k}, \frac{k}{n-k} \ln k\right\}\right)$, which asymptotically improves the best known approximation guarantee for directed graphs for all values of $n, k$, and for undirected graphs for $k>\sqrt{n / 6}$. Moreover, this is the first algorithm that has an approximation guarantee better than $\Theta(k)$ for all values of $n, k$. Our approximation ratio also provides an upper bound on the integrality gap of the standard LP-relaxation to the problem.

As a byproduct, we also get the following result which is of independent interest. To get a faster implementation of our algorithms, we consider the problem of adding a minimumcost edge set to increase the outconnectivity of a directed graph by $\Delta$; a graph is said to be $\ell$-outconnected from its node $r$ if it contains $\ell$ internally disjoint paths from $r$ to any other node. The best known time complexity for the later problem is $O\left(\mathrm{~m}^{3}\right)$. For the particular case of $\Delta=1$, we give a primal-dual algorithm with running time $O\left(\mathrm{~m}^{2}\right)$.
\end{abstract}

\section{Categories and Subject Descriptors}

F.2.2 [Theory of computation]: Analysis of Algorithms

Permission to make digital or hard copies of all or part of this work for personal or classroom use is granted without fee provided that copies are not made or distributed for profit or commercial advantage and that copies bear this notice and the full citation on the first page. To copy otherwise, to republish, to post on servers or to redistribute to lists, requires prior specific permission and/or a fee.

STOC'04, June 13-15, 2004, Chicago, Illinois, USA.

Copyright 2004 ACM 1-58113-852-0/04/0006 ...\$5.00.

\section{General Terms}

Algorithms

\section{INTRODUCTION AND PRELIMINARIES}

A basic problem in network design is given a graph to find its minimum cost $k$-connected spanning subgraph; a graph is $k$ (-node) connected if it is simple and there are at least $k$ internally disjoint paths from every node to the other. This problem is NP-hard for undirected graphs with $k=2$, and for directed graphs with $k=1$. The best known approximation guarantees for this problem were $O\left(\min \left\{k, \frac{n}{\sqrt{n-k}}\right\}\right)$ for both directed and undirected graphs $[13,4]$, and $O(\ln k)$ for undirected graphs with $n \geq 6 k^{2}$ [4], where $n$ is the number of nodes in the input graph. (For undirected graphs, Ravi and Williamson [24] claimed an $O(\ln k)$-approximation algorithm, but the proof was found to contain an error, see [25].) Better approximation guarantees are known for restricted edge costs, as follows. For metric costs: $2+2(k-$ $1) / n$ for undirected graphs [14] (for a slight improvement to $2+(k-1) / n$ see [13]) and $2+k / n$ for directed graphs [13]. For uniform costs there is a $(1+1 / k)$-approximation algorithm for both directed and undirected graphs [3]. The case when the input graph is complete and the cost of every edge is in $\{0,1\}$ (so called "vertex-connectivity augmentation problem") is polynomially solvable for directed graphs [6]; polynomial algorithms that compute a near optimal solution for undirected graphs are given in [10, 12]. But in this paper we consider the case of general costs only.

The main result of this paper is the following theorem:

THEOREM 1.1. There exists an algorithm for the minimumcost $k$-connected subgraph problem with approximation ratio $O\left(\ln k \cdot \min \left\{\sqrt{k}, \frac{k}{n-k} \ln k\right\}\right)$ and running time $O\left(n^{5} m\right)$.

This gives the first algorithm that has an approximation guarantee better than $\Theta(k)$ for all values of $n, k$, and improves the previously best known approximation guarantees for directed graphs for all values of $n, k$, and for undirected graphs for $k>\sqrt{n / 6}$. Note that our approximation ratio is $O\left(\ln ^{2} k\right)$ except for very large values of $k$ (namely, $k=n-o(n))$. In particular, for instances with $n>k c$ where $c>1$ is a fixed constant, the approximation ratio is $O\left(\ln ^{2} k\right)$. For example, the previously best approximation ratios for $k=\sqrt{n}$ and $k=n / 2$ were $O(k)$ and $O(\sqrt{k})$, respectively; our approximation ratio for both these cases is 
$O\left(\ln ^{2} k\right)$. For $k=n-o(n)$ the improvement is from $O(k)$ to $O(\sqrt{k} \ln k)$. Our algorithm is also significantly faster than the $O\left(\frac{n}{\sqrt{n-k}}\right)$-approximation algorithm of [4] which requires solving linear programs with the ellipsoids method. Moreover, our algorithm is combinatorial in the sense that it does not have to deal with numerical precision issues of maintaining sufficiently large number of bits in computations (such as taking square roots or computing volumes) so as to avoid errors.

Our $O\left(\frac{k}{n-k} \ln ^{2} k\right)$-approximation algorithm is based on a new result on $\ell$-connected $p$-critical graphs which is of independent interest in graph theory. Namely, we will prove that any $\ell$-connected graph (directed or not) on $n$ nodes has a subset $U$ of nodes with $|U|=O\left(\frac{\ell}{n-\ell} \ln \ell\right)$ such that no node-cut of cardinality $\ell$ contains $U$; we call such $U$ an $\ell$-cover (since $U$ covers the complementaries of node-cuts of cardinality $\ell$ ), and denote by $\tau_{\ell}(G)$ the minimum cardinality of an $\ell$-cover in $G$. An $\ell$-connected graph $G$ is $p$-critical if $p<\tau_{\ell}(G)$ (this definition is shown to be equivalent to the one used in the papers on the topic, see Section 1.2). For undirected graphs, our result partly bridges the gap between two main bounds: the obvious fact that $\tau_{\ell}(G) \leq \ell+1$ and a result of Mader [18] which states that $\tau_{\ell}(G) \leq 3$ for $n \geq 6 \ell^{2}$. Other previous bounds were for undirected graphs only, and of the type $\tau_{\ell}(G) \leq \Theta(\ell)$ (e.g., [15]), or of the type $\tau_{\ell}(G) \leq \Theta(1)$ for $n=\bar{\Omega}\left(\ell^{2}\right)$ (e.g., [19]). Our result gives the first nontrivial bound in the intermediate range for undirected graphs, and overall the first nontrivial bound for directed graphs. Moreover, our proof provides a polynomial algorithm that computes an $\ell$-cover within the stated bound.

Throughout the paper, let $\mathcal{G}=(V, \mathcal{E})$ denote the input graph with nonnegative costs on the edges; $n$ denotes the number of nodes in $\mathcal{G}$, and $m$ the number of edges in $\mathcal{G}$. Unless stated otherwise, "graph" stands for both directed and undirected graph.

This paper is organized as follows. In the rest of this section we will introduce a standard LP-relaxation to the mincost $k$-connected subgraph problem, and state some simple facts about $\ell$-outconnected graphs and $p$-critical graphs. In Sections 2 and 3 we give our algorithm for the min-cost $k$ connected subgraph problem: in Section 2 we establish existence of an $\ell$ cover of size $O\left(\frac{\ell}{n-\ell} \ln \ell\right)$ and show how to compute it, which implies an $O\left(\frac{\ell}{n-\ell} \ln ^{2} \ell\right)$-approximation algorithm, while Section 3 present our primal-dual $O(\sqrt{n} \ln k)$ approximation algorithm. In Section 4 we present a fast primal-dual algorithm for increasing the outconnectivity of a directed graph by 1 .

\subsection{LP-relaxation and $\ell$-outconnected graphs}

For an edge set or a graph $J$ on a node set $V$ and $S, T \subseteq V$ let $\delta_{J}(S, T)$ denote the set of edges in $J$ going from $S$ to $T$. By Menger's Theorem, there are $k$ internally disjoint paths from a node $s$ to a node $t$ in a graph $G=(V, E)$ if and only if $\left|\delta_{E}(S, T)\right| \geq k-(n-|S \cup T|)$ for all disjoint $S, T \subset V$ with $s \in S$ and $t \in T$. We will compare the cost of our solutions to the optima opt $_{k}$ of the following LP-relaxation for the minimum cost $k$-node connected spanning subgraph that has been introduced in [6] and used in [4]:

$$
\begin{array}{ll}
\text { opt }_{k}= & \min \sum_{e \in \mathcal{E}} c_{e} x_{e} \\
\text { s.t. } & \sum_{e \in \delta_{\mathcal{E}}(S, T)} x_{e} \geq k-(n-|S \cup T|) \\
& \forall \emptyset \neq S, T \subset V, S \cap T=\emptyset \\
& 0 \leq x_{e} \leq 1 \quad \forall e \in \mathcal{E} .
\end{array}
$$

A graph is said to be $\ell$-outconnected from a node $r$ if it contains $\ell$ internally disjoint paths from $r$ to any other node; a graph is $\ell$-inconnected to $r$ if its reverse graph is $\ell$-outconnected from $r$ (for undirected graphs these two concepts mean the same). Frank and Tardos [7] showed that for directed graphs, the problem of finding an $\ell$-outconnected spanning subgraph of minimum cost is solvable in polynomial time; a faster algorithm with time complexity $O\left(\ell^{2} n^{2} m\right)=$ $O\left(m^{3}\right)$ is due to Gabow [8] (observe that $n \ell=O(m)$ in an $\ell$-outconnected graph).

Let $G=(V, E)$ be an $\ell$-connected spanning subgraph of cost zero of a directed graph $\mathcal{G}$, and suppose that $G$ has a subset $U$ of nodes such that no node-cut of cardinality $\ell$ contains all of them. Then using the algorithm of [7] it is easy to get a $2|U|$-approximation algorithm for the problem of augmenting $G$ to be $(\ell+1)$-connected by adding an edge set of minimum cost: for each root $r \in U$ we compute an $(\ell+1)$-outconnected and $(\ell+1)$-inconnected spanning subgraph and take the union of these $2|U|$ subgraphs. In fact, the following lemma, which can be easily deduced from $[5$, Theorem 7] (e.g, see [4, Lemma 3.4]) implies that the augmenting edge set produced has cost at most $\frac{2|U|}{k-\ell} o p t_{k}$.

LEMMA 1.2. Let $G$ be an $\ell$-outconnected from $r$ subgraph of cost zero of a directed graph $\mathcal{G}$, and for an integer $p$ let $\alpha^{p}$ be the minimum cost of an $(\ell+p)$-outconnected spanning subgraph of $\mathcal{G}$. Then $\alpha^{1} \leq \alpha^{p} / p$. In particular, for $\ell<k$ the minimum cost of an $(\ell+1)$-outconnected spanning subgraph of $\mathcal{G}$ is at most $\frac{1}{k-\ell}$ opt $_{k}$.

Khuller and Raghavachari [14] observed that the algorithm of [7] implies a 2-approximation algorithm for the problem of finding an optimal $\ell$-outconnected subgraph of an undirected graph, as follows. First, replace every undirected edge $e$ of $\mathcal{G}$ by the two antiparallel directed edges with the same ends and of the same cost as $e$. Then compute an optimal $\ell$-outconnected from $r$ subdigraph and output its underlying (undirected) simple graph. Several papers used these observations for designing approximation algorithms for node connectivity problems, e.g., see [1, 2, 13, 4].

\section{$1.2 p$-critical graphs and $k$-connected subgraphs}

Let $\kappa(G)$ denote the connectivity of $G$, that is the maximum integer $\ell$ for which $G$ is $\ell$-connected. A graph $G=$ $(V, E)$ is $p$-critical if $\kappa(G-U)=\kappa(G)-|U|$ for any $U \subset V$ with $|U| \leq p$. One can characterize $p$-critical graphs in terms of covers of set families, as follows. Let $X^{*}=X_{G}^{*}=\{v \in$ $\left.V-X: \delta_{G}(X, v)=\emptyset\right\}$ denote the "node complement" of $X$ in $G$. We say that $X \subset V$ is an $\ell$-fragment if $X^{*} \neq \emptyset$ and $\left|V-\left(X \cup X^{*}\right)\right|=\ell$. Let $G$ be an $\ell$-connected graph. Note that Menger's Theorem implies the following well known statement:

Proposition 1.3. A noncomplete $\ell$-connected graph $G$ is $(\ell+1)$-connected if and only if $G$ has no $\ell$-fragments. 
Given a family $\mathcal{F}$ of subsets of a groundset $V$ we say that $U \subseteq V$ covers $\mathcal{F}$ if $U$ intersect every set in $\mathcal{F}$. Let $\mathcal{F}_{\ell}(G)$ be the family of all $\ell$-fragments of $G$. We say that $U \subseteq V$ is an $\ell$-cover of $G$ if $U$ covers $\left\{X \cup X^{*}: X \in \mathcal{F}_{\ell}(G)\right\}$; let $\tau_{\ell}(G)$ denote the minimum cardinality of an $\ell$-cover of $G$. From Proposition 1.3 we have:

Proposition 1.4. Let $G=(V, E)$ be a noncomplete graph with $\kappa(G)=\ell$. Then:

(i) $G$ is $p$-critical if and only if for any $U \subseteq V$ with $|U| \leq p$ there exists an $\ell$-fragment $X$ with $U \cap\left(X \cup X^{*}\right)=\emptyset$. Thus if $G$ is $p$-critical then $G$ is $p^{\prime}$-critical for any $p^{\prime} \leq p$, and $\tau_{\ell}(G)-1$ is the maximum $p$ for which $G$ is $p$-critical.

(ii) $U$ is an $\ell$-cover of $G$ if and only if there exists an edge set $F$ incident to $U$ such that $G+F$ is $(\ell+1)$-connected.

Combining Proposition 1.4(ii) with Lemma 1.2 it is not hard to get the following statement, which was implicitly proved in [4] for undirected graphs.

Proposition 1.5 ([4]). Suppose that there is a polynomial algorithm that finds in any $\ell$-connected graph $G$ on $n$ nodes an $\ell$-cover of $G$ of size at most $t(\ell, n)$. Then there exists a polynomial algorithm that for instances of the minimum $k$-connected subgraph problem on $n$ nodes finds a feasible solution of cost at most opt ${ }_{k} \cdot 2 \sum_{\ell=0}^{k-1} \frac{t(\ell, n)}{k-\ell}=$ opt $_{k}$. $O\left(\ln k \cdot \max _{0 \leq \ell \leq k-1} t(\ell, n)\right)$.

For undirected graphs with $n \geq 6 k^{2}$ Cheriyan et. al. [4] gave a $6 H(k)$-approximation algorithm for the undirected min-cost $k$-connected subgraph problem combining Proposition 1.5 with the following theorem due to Mader:

TheOrem 1.6 ([18]). Any undirected 3-critical graph $G$ has less than $6 \kappa(G)^{2}$ nodes.

In a recent paper [19] Mader improved his bound for 3critical graphs to $n \leq \kappa(G)(2 \kappa(G)-1)$; hence via Proposition 1.5 the $6 H(k)$-approximation algorithm of [4] is valid for $n \geq k(2 k-1)$ as well.

On the other hand, it is easy to see that there are no $\kappa(G)$ critical non-complete graphs. But for undirected graphs, a stronger result was conjectured in [23], and answered by Su:

TheOREM 1.7 ([26]). If a noncomplete graph $G$ is $p$ critical, then $p \leq\lfloor\kappa(G) / 2\rfloor$.

For a survey on $p$-critical graphs see [21, 22], and for some recent results see $[19,20]$ and $[15]$.

\section{COMPUTING LOGARITHMIC COVERS}

Note that in terms of covers of set families Theorem 1.6 states that for an undirected graph $G$ with $\kappa(G)=\ell$ holds $\tau_{\ell}(G) \leq 3$ if $n \geq 6 \ell^{2}$, and Theorem 1.7 states that $\tau_{\ell}(G) \leq$ $\lfloor\ell / 2\rfloor+1$ (if $n \geq \ell+2$ ). Our result on $p$-critical graphs partly bridges the gap between these two bounds, and also gives the first nontrivial bound on $\tau_{\ell}(G)$ for directed graphs. Let $\theta=\theta(\ell, n)=\frac{2 n}{n+\ell}$.

THEOREM 2.1. There exists a polynomial algorithm that given a noncomplete $\ell$-connected graph $G$ on $n$ nodes finds an $\ell$-cover of $G$ of size at most

$t(\ell, n)=5+\frac{3 \ell}{n-\ell}+\frac{1}{\ln \theta} \ln \frac{1}{2}\left(\ell-1-\ell^{2} / n\right)=O\left(\frac{\ell}{n-\ell} \ln \ell\right)$ if $G$ is undirected, and of size at most $2 t(\ell, n)$ if $G$ is directed.

Combining with Proposition 1.5 we get:

TheOREM 2.2. For the minimum cost $k$-connected subgraph problem there exists a polynomial algorithm that finds a feasible solution of cost at most opt ${ }_{k} \cdot O\left(\frac{k}{n-k} \ln ^{2} k\right)$.

Remark: Note that $\tau_{\ell}(G)$ is the minimum cardinality of a cover of the corresponding $(n-\ell)$-uniform hypergraph. Several general bounds on covers of uniform hypergraphs are known, e.g., see [9]. But, as far as we can see, none of them implies the bound given in Theorem 2.1.

We need several definitions and simple facts to prove Theorem 2.1. Let $\ell$ be a fixed integer, and let $G$ be a graph with $\kappa(G) \geq \ell$. An $\ell$-fragment $X$ of $G$ is small if $|X| \leq\left|X^{*}\right|$, that is if $|X| \leq\lfloor(n-\ell) / 2\rfloor$. Note that by Proposition $1.3 G$ is $(\ell+1)$-connected if, and only if, $G$ (and the reverse graph of $G$, if $G$ is directed) has no small $\ell$ fragments. Let $\mathcal{S}_{\ell}(G)$ denote the family of all small $\ell$-fragments of $G$. The following Lemma is well known, e.g., see [10, Lemma 1.2], where it was stated for undirected graphs.

Lemma 2.3. Let $X, Y$ be two intersecting $\ell$-fragments in an $\ell$-connected (directed or undirected) graph $G$ on $n$ nodes. If $n-|X \cup Y| \geq \ell$ then $X \cap Y$ is an $\ell$-fragment, and if a strict inequality holds, then also $X \cup Y$ is an $\ell$-fragment. In particular, the intersection of two intersecting small $\ell$ fragments is also a small $\ell$-fragment.

A core of $G$ is an inclusion minimal small $\ell$-fragment. By Lemma 2.3 the cores of $G$ are pairwise disjoint, and let $\nu(G)=\nu_{\ell}(G)$ denote their number; note that if $\kappa(G)>\ell$ then $\nu_{\ell}(G)=0$. For a core $C_{i}$ of $G$, let $A_{i}$ be the the union of all small $\ell$-fragments that contain a unique core which is $C_{i}$. Let $\mathcal{A}_{\ell}(G)=\left\{A_{1}, \ldots, A_{\nu(G)}\right\}$. The properties of the sets in $\mathcal{A}$ that we use are summarized in the following statement:

Corollary 2.4. The sets in $\mathcal{A}_{\ell}(G)$ are pairwise disjoint. Moreover, for every $A \in \mathcal{A}_{\ell}(G)$ holds: either $A$ is an $\ell$ fragment, or $|A| \geq n-\ell$ (and $\left.A^{*}=\emptyset\right)$; thus $\left|A \cup A^{*}\right| \geq n-\ell$.

Proof. Suppose to the contrary that $A_{i}$ and $A_{j}$ intersect for some $1 \leq i \neq j \leq \nu(G)$. Then, by the definition of $A_{i}, A_{j}$, there are two small $\ell$-fragments $D_{i}, D_{j}$ such that: $D_{i}$ contains a unique core which is $C_{i}, D_{j}$ contains a unique core which is $C_{j}$, and such that $D_{i}, D_{j}$ intersect. By Lemma 2.3 $D_{i} \cap D_{j}$ is a small $\ell$-fragment, and thus contains a core $C$. This implies that $D_{i}$ contains the two cores $C_{i}$ and $C$, which gives a contradiction.

To prove the second statement, let us fix some set $A \in$ $\mathcal{A}_{\ell}(G)$. Since the sets in $\mathcal{A}_{\ell}(G)$ are disjoint, $A$ contains a unique core, say $C$. Consider the family $\mathcal{D}$ of all small $\ell-$ fragments that contain a unique core which is $C$, so $A$ is the union of the sets in $\mathcal{D}$. If $n-|A| \leq \ell$, then clearly $\left|A \cup A^{*}\right| \geq|A| \geq n-\ell$ (in fact, in this case $A^{*}=\emptyset$, and thus $\left.\left|A \cup A^{*}\right|=|A|\right)$. Otherwise, $n-|A| \geq \ell+1$; then by Lemma 2.3 , the union of the sets in $\mathcal{D}$ is an $\ell$-fragment, and thus $\left|A \cup A^{*}\right|=n-\ell$. In both cases, the statement is valid.

Note that Corollary 2.4 does not imply that the sets in $\mathcal{A}$ are small, or that they are $\ell$-fragments; it might be that $A$ is large and that $A^{*}=\emptyset$ for some $A \in \mathcal{A}$, but in any case, $\left|A \cup A^{*}\right| \geq n-\ell$ holds. 
Lemma 2.5. Let $\mathcal{A}$ be a family of set on a groundset $V$ such that for every $A \in \mathcal{A}$ holds $|A| \geq n-\ell$, where $n=|V|$ and $\ell$ is an integer. Then there exists an element $r \in V$ that covers (that is, intersects) at least $\left(1-\frac{\ell}{n}\right)|\mathcal{A}|$ sets in $\mathcal{A}$.

Proof. For $r \in V$, let $\mathcal{A}_{r}=\{A \in \mathcal{A}: r \in A\}$ be the sets in $\mathcal{A}$ covered by $r$. The claim follows since we have

$$
\sum_{r \in V}\left|\mathcal{A}_{r}\right|=\sum_{A \in \mathcal{A}}|A| \geq|\mathcal{A}|(n-\ell) .
$$

For $r \in V$ let $F_{r}=\{v r: v \in V-r\}$, and let $G+F_{r}$ be the graph obtained by adding an edge from every node $v \in V$ to $r$, if such does not exist in $G$. We say that $r \in V$ outercovers $A \in \mathcal{A}_{\ell}(G)$ if $r \in A^{*}$.

Lemma 2.6. Let $C_{i}$ be a core of $G$. If $r$ outercovers $A_{i}$ then any small $\ell$-fragment $X$ of $G+F_{r}$ that contains $C_{i}$ must contain a core of $G$ distinct from $C_{i}$.

Proof. Let $X$ be a small $\ell$-fragment of $G+F_{r}$ that contains $C_{i}$. Assume by contradiction that this is the unique core of $G$ that $X$ contains. Note that $X$ is a small $\ell$-fragment of $G$. Since $A_{i}$ is defined as the union of all small $\ell$-fragments of $G$ containing $C_{i}$ as their unique core, we obtain that $X \subseteq A_{i}$. This gives a contradiction, since then $r \in A_{i}^{*} \subseteq X^{*}$, which implies that $X$ cannot be an $\ell$-fragment of $G+F_{r}$.

LEMMA 2.7. Let $r$ be a node that outercovers $q$ sets in $\mathcal{A}_{\ell}(G)$. Then $\nu_{\ell}\left(G+F_{r}\right) \leq \nu_{\ell}(G)-q / 2$.

Proof. If $\kappa\left(G+F_{r}\right)>\ell$ then $\nu_{\ell}\left(G+F_{r}\right)=0$ and the statement is obvious, so assume that $\kappa\left(G+F_{r}\right)=\ell$. By Lemma 2.3, the cores of $G+F_{r}$ are pairwise disjoint. Clearly, every core of $G+F_{r}$ is a small $\ell$-fragment of $G$, and thus contains at least one core of $G$. Let $t$ be the number of cores of $G+F_{r}$ containing exactly one core of $G$. By Lemma 2.6, any core $C$ of $G+F_{r}$ that contains some core $C_{i}$ of $G$ with $r \in A_{i}^{*}$ must contain another core of $G$ distinct from $C_{i}$, so such $C$ contains at least two cores of $G$. Thus $t \leq \nu_{\ell}(G)-q$. From this we get that $\nu_{\ell}\left(G+F_{r}\right) \leq t+\left(\nu_{\ell}(G)-t\right) / 2 \leq$ $\nu_{\ell}(G)-q / 2$, as required.

Since the sets in $\mathcal{A}_{\ell}(G)$ are pairwise disjoint, a node can belong to at most one of them. Thus, if $\mathcal{A}^{\prime} \subseteq \mathcal{A}_{\ell}(G)$ and $r$ covers $\left\{A \cup A^{*}: A \in \mathcal{A}^{\prime}\right\}$, then there is most one set $A^{\prime} \in \mathcal{A}^{\prime}$ such that $r \in A^{\prime}$; for any other $A \in \mathcal{A}-A^{\prime}$ we must have $r \in A^{*}$, hence $r$ outercovers at least $\left|\mathcal{A}^{\prime}\right|-1$ sets in $\mathcal{A}^{\prime}$. Combining this with Corollary 2.4 and Lemmas 2.5 and 2.7 we get:

Corollary 2.8. Any $\ell$-connected graph $G$ has a node $r$ that outercovers at least $\nu_{\ell}(G)(1-\ell / n)-1$ sets in $\mathcal{A}_{\ell}(G)$, and $\nu_{\ell}\left(G+F_{r}\right) \leq \frac{n+\ell}{2 n} \nu_{\ell}(G)+1 / 2$.

Let us apply the following algorithm on an $\ell$-connected graph $G$ starting with $U=\emptyset$.

While $\nu_{\ell}(G)>0$ do:

1. Find a node $r$ for which $\nu_{\ell}\left(G+F_{r}\right)$ is minimal;

2. $U \leftarrow U+r, G \leftarrow G+F_{r}$;
End While

Output $U$.

By Proposition 1.4(ii), at the end of the algorithm $U$ is an $\ell$-cover, and let us estimate its size. Let $t_{j}$ be the number of cores in $G$ after $j$ iterations of the main loop, and set $\theta=\frac{2 n}{n+\ell}$ and $\alpha=1 / \theta$. Corollary 2.8 gives the recursive bound

$$
t_{j+1} \leq \alpha t_{j}+1 / 2
$$

We will prove later that $t_{1} \leq \ell$ (see Corollary 2.10 below), which implies:

$$
\begin{aligned}
t_{j} & \leq \alpha^{j-1} \ell+\frac{1}{2}\left(1+\alpha+\cdots+\alpha^{j-2}\right) \\
& =\alpha^{j-1} \ell+\frac{1-\alpha^{j-1}}{2(1-\alpha)} \\
& =\alpha^{j-1}\left(\ell-\frac{1}{2(1-\alpha)}\right)+\frac{1}{2(1-\alpha)} \\
& =\frac{1}{\theta^{j-1}}\left(\ell-\frac{n}{n-\ell}\right)+\frac{n}{n-\ell} .
\end{aligned}
$$

Let $\beta=3 n /(n-\ell)=3+3 \ell /(n-\ell)$. Easy computations show that

$$
t_{j} \leq \beta \quad \text { for } \quad j \geq j(\beta) \equiv \frac{1}{\ln \theta} \ln \frac{1}{2}\left(\ell-1-\ell^{2} / n\right)+1 .
$$

On the other hand, if $t_{j}>0$ then $t_{j+1} \leq t_{j}-1$ since $\nu_{\ell}(G+$ $\left.F_{r}\right) \leq \nu_{\ell}(G)-1$ for any node $r$ belonging to a core $C_{i}$ of $G$ (indeed, every core of $G+F_{r}$ must contain some core of $G$, but cannot contain $C_{i}$ ). Thus the number of iterations in the algorithm (which equals to the size of the cover found) is bounded by $\lceil j(\beta)\rceil+\lfloor\beta\rfloor \leq t(\ell, n)$. This proves Theorem 2.1 for undirected graphs. In the case of a directed graph $G$, we apply the above procedure twice: on $G$ and on the reverse graph of $G$, and take the union of the resulting two node sets.

Let us now analyze the time complexity of our approximation algorithm for $k$-connected spanning subgraphs. We omit the details, but note that using max-flow techniques an $\ell$-cover as in Theorem 2.1 it can be found in $\ell^{2} m n$ time. We also need to find a minimum-cost edge set to increase the outconnectivity from $\ell$ to $\ell+1$ from each node in the cover found. In Section 4 we will discuss a primal dual algorithm for this restricted problem, and show that it can be implemented in $O\left(\mathrm{~m}^{2}\right)$ time, see Theorem 4.1 to follow. As the size of the cover found is $O(\ell)$, we get that the overall time complexity for increasing connectivity from $\ell$ to $\ell+1$ is $O\left(\ell^{2} m n+\ell m^{2}\right)=O\left(\ell m^{2}\right)$, where $m \geq n \ell$ is the number of edges in $\mathcal{G}$. Consequently, the overall running time of the algorithm is $O\left(k^{2} m^{2}\right)=O\left(n^{4} m\right)$.

Let us now show that $t_{1} \leq \ell$, and discuss some consequences from our approach. The following statement is obvious.

Lemma 2.9. Let $r$ be a node of a (directed or undirected) noncomplete graph $G=(V, E)$ with $\kappa(G)=\ell$, and let $N_{r}=$ $\{v \in V:$ vr $\in E\}$ be the nodes in $G$ having $r$ as their neighbor. Then $N_{r}$ covers all $\ell$-fragments of $G+F_{r}$.

An $\ell$-connected graph $J$ is minimally $\ell$-connected if $J-e$ is not $\ell$-connected for every edge $e$ of $J$. Mader $[16,17]$ showed that any minimally $\ell$-connected graph $J$ on $n$ nodes has at least $\frac{(\ell-1) n+2}{2 \ell-1}$ nodes of degree (indegree, if $J$ is directed) 
$\ell$ each. Since for any $\ell$-connected spanning subgraph $J$ of an $\ell$-connected graph $G$ holds $\mathcal{F}_{\ell}(G) \subseteq \mathcal{F}_{\ell}(J)$, Lemma 2.9 implies the following corollary, which also proves that $t_{1} \leq \ell$.

Corollary 2.10. Let $G=(V, E)$ be an $\ell$-connected graph. Then there is $R \subseteq V$ with $|R| \geq \frac{(\ell-1) n+2}{2 \ell-1}$ such that for any $r \in R$ holds: $r$ and at most $\ell$ nodes having $r$ as their neighbor cover all $\ell$-fragments of $G$, and in particular $\nu_{\ell}\left(G+F_{r}\right) \leq \ell$.

We note that for a directed graph $G$ Corollary 2.10 implies only the trivial bound $\tau_{\ell}(G) \leq \ell+1$; however for undirected $G$ the following Theorem provides an easy proof of Theorem 1.7, which is similar to the one given by Jordán in [11]; recall that in terms of covers Theorem 1.7 states that $\tau_{\ell}(G) \leq\lfloor\ell / 2\rfloor+1$.

THEOREM 2.11. Let $G$ be an undirected $\ell$-connected graph and let $W$ be a cover of $\mathcal{F}_{\ell}(G)$. Then there exists an $\ell$-cover $U \subseteq W$ of size at most $\lfloor|W| / 2\rfloor$.

Proof. In [16] Mader implicitly proved (e.g., see [10] and [13, Corollary 2.2]) that if $W$ covers all the $\ell$-fragments of an undirected $\ell$-connected graph $G$ then there exists a forest $F$ on $W$ such that $G+F$ is $(\ell+1)$-connected. Since $F$ is a forest, there exists $U \subseteq W$ such that $|U| \leq\lfloor|W| / 2\rfloor$ and every edge in $F$ is incident to a node in $U$. Thus, by Proposition 1.4 (ii), $U$ is a cover of $\mathcal{F}_{\ell}(G)$ as required.

\section{PRIMAL-DUAL ALGORITHM}

In this section we prove the following theorem:

THEOREM 3.1. For the problem of making a $k_{0}$-connected graph (directed or undirected) $k$-connected by adding a minimumcost edge set there exists an approximation algorithm with approximation ratio $O\left(\sqrt{n} H\left(k-k_{0}\right)\right)=O(\sqrt{n} \ln k)$ and time complexity $O\left(k m\left(k^{2} n^{2}+\sqrt{n} m\right)\right)=O\left(n^{5} m\right)$.

We start by giving an algorithm for increasing the connectivity of a directed graph by one. Let $G=(V, E)$ be an $\ell$ connected spanning subgraph of a directed graph $\mathcal{G}=(V, \mathcal{E})$, such that all the edges in $E$ have cost zero, and let $I=\mathcal{E}-E$. Let $\mathcal{S}=\mathcal{S}_{\ell}(G)$ denote the set of small $\ell$-fragments of $G$. For an edge set $F$ and $S \in \mathcal{S}$, let $d_{F}(S)=\left|\delta_{F}\left(S, S^{*}\right)\right|$ be the number of edges in $F$ going from $S$ to $S^{*}$.

Recall that $G+F$ is $(\ell+1)$-connected if and only if the graph $G+F$ and its reverse graph have no small $\ell$-fragments. Note that for $F \subseteq I=\mathcal{E}-E, G+F$ has no small $\ell$-fragments if and only if $d_{F}\left(S, S^{*}\right) \geq 1$ for any $S \in \mathcal{S}$. Consider the following linear program $(\mathrm{P})$ and its dual program $(\mathrm{D})$, where $(\mathrm{P})$ is a linear relaxation for the problem of finding a minimum cost augmenting edge set $F$ such that $G+F$ contains no small $\ell$-fragments:

The primal programm:

$$
\begin{array}{lll}
\min & \sum_{e \in I} c_{e} x_{e} \\
\text { s.t. } & \sum_{\substack{e \in \delta_{I}\left(S, S^{*}\right) \\
x_{e} \geq 0}} x_{e} \geq 1 \quad \forall S \in \mathcal{S} \\
& \forall e \in I
\end{array}
$$

And the dual programm:

$$
\begin{array}{ll}
\max & \sum_{S \in \mathcal{S}} y_{S} \\
\text { s.t. } & \sum_{\substack{S \in \mathcal{S}: e \in \delta_{I}\left(S, S^{*}\right) \\
y_{S} \geq 0}} y_{S} \leq c_{e} \quad \forall e \in I \\
& \forall S \in \mathcal{S} .
\end{array}
$$

LEMma 3.2. Let $x$ be an optimal solution to $(P)$. Then $\sum_{e \in I} c_{e} x_{e} \leq \frac{1}{k-\ell} o p t_{k}$.

Proof. Let $x$ be an optimal solution to the LP-relaxation for the min-cost $k$-connected spanning subgraph problem (given in Section 1.1). Define $x_{e}^{\prime}=1$ if $e \in E$ and $x_{e}^{\prime}=$ $\frac{1}{k-l} x_{e}$ otherwise. Then $x^{\prime}$ is a feasible solution to $(\mathrm{P})$. Since all the edges in $E$ have cost zero, the claim follows.

Given a feasible solution $y$ to (D), an edge $e \in I$ is tight if the corresponding inequality in the dual program (D) holds with equality. If $F^{+} \subseteq I$ is a set of tight edges, then

$$
c\left(F^{+}\right)=\sum_{e \in F^{+}} c_{e}=\sum_{e \in F} \sum_{S \in \mathcal{S}: e \in \delta\left(S, S^{*}\right)} y_{S}=\sum_{S \in \mathcal{S}} d_{F}(S) y_{S} .
$$

Recall that by Lemma 2.3 the cores of $G$ are disjoint. Let us fix the threshold $\beta=\sqrt{2 n}$ and apply the following procedure:

\section{Procedure 1:}

While $\nu_{\ell}(G) \geq \beta$, raise dual variables corresponding to cores of $G$ uniformly until some edge $e \in I$ becomes tight, and add this edge to $G$.

Let $\tilde{F}^{+}$be the set of edges added to the input graph $G$ by Procedure 1.

Lemma 3.3. Let $F^{+} \subseteq \tilde{F}^{+}$. Then $c\left(F^{+}\right) \leq \frac{\left|F^{+}\right|}{\beta} \frac{o p t_{k}}{k-\ell}$.

Proof. Let $y$ be the dual solution produced by Procedure 1. Since the edges in $F^{+}$are tight, we have $c\left(F^{+}\right)=$ $\sum_{S \in \mathcal{S}} d_{F^{+}}(S) y_{S}$, by (1). Let $\mathcal{C}_{i}$ be the family of cores of $G$ at iteration $i$, and let $\varepsilon_{i}$ be the amount at which they were raised at iteration $i, i=1, \ldots, q$. Note that for any set $S \in \mathcal{S}$ holds $y_{S}=\sum_{S \in \mathcal{C}_{i}} \varepsilon_{i}$. Using this, together with the fact that the sets in $\mathcal{C}_{i}$ are disjoint and that $\left|\mathcal{C}_{i}\right| \geq \beta$ we get:

$$
\begin{aligned}
\sum_{S \in \mathcal{S}} d_{F^{+}}(S) y_{S} & =\sum_{i=1}^{q} \varepsilon_{i} \sum_{S \in \mathcal{C}_{i}} d_{F^{+}}(S) \\
& \leq \sum_{i=1}^{q} \varepsilon_{i}\left|F^{+}\right| \frac{\left|\mathcal{C}_{i}\right|}{\beta} \\
& =\frac{\left|F^{+}\right|}{\beta} \sum_{i=1}^{q} \varepsilon_{i}\left|\mathcal{C}_{i}\right| \\
& =\frac{\left|F^{+}\right|}{\beta} \sum_{S \in \mathcal{S}} y_{S} \\
& \leq \frac{\left|F^{+}\right|}{\beta} \frac{o p t^{*}}{k-\ell} .
\end{aligned}
$$

The first inequality follows by upper bounding 1 by $\left|\mathcal{C}_{i}\right| / \beta$, and noting that in $\sum_{S \in \mathcal{C}_{i}} d_{F^{+}}(S)$ every edge is counted exactly once. The last inequality follows from Lemma 3.2 and the Weak Duality Theorem. 
After executing Procedure 1 , let $C_{1}, \ldots, C_{\nu^{+}}$be the cores of $G+\tilde{F}^{+}$.

Procedure 2:

For $j=1, \ldots, \nu^{+}$, choose $r_{j} \in C_{j}$, and compute an optimal edge set $\tilde{F}_{j}^{+}$such that $G+\tilde{F}_{j}^{+}$is $(\ell+1)$-outconnected from $r_{j}$.

Note that by Lemma $1.2 c\left(\tilde{F}_{j}\right) \leq \frac{1}{k-\ell} o p t_{k}, j=1, \ldots, \nu^{+}$.

We then apply Procedures 1 and 2 on the reverse graph of $G+I$ to find appropriate edge sets $\tilde{F}^{-}$and $\tilde{F}_{1}^{-}, \ldots, \tilde{F}_{\nu^{-}}^{-}$. Let $\tilde{F}$ be the union of all the edge sets found. Then $G+\tilde{F}$ is $(\ell+1)$-connected. The last step in our algorithm is finding an inclusion minimal edge set $F \subseteq \tilde{F}$ such that $G+F$ is $(\ell+1)$-connected. Note that $|\tilde{F}|$ might be large, but the following statement shows that $|F|=O(n)$.

THEOREM 3.4 ([17]). Let $G$ be an $\ell$-connected directed graph, and let $F$ be an inclusion minimal augmenting edge set such that $G+F$ is $(\ell+1)$-connected. Then $|F| \leq 2 n-1$.

LEMma 3.5. The algorithm produces a feasible solution of cost at most $\frac{4 \sqrt{2 n}}{k-\ell}$ opt $_{k}$.

Proof. By Theorem 3.4, $|F| \leq 2 n$. Set $F^{+}=\tilde{F}^{+} \cap F$, $F^{-}=\tilde{F}^{-} \cap F, F_{j}^{+}=\tilde{F}_{j}^{+} \cap F$ for $j=1, \ldots, \nu^{+}$, and $F_{j}^{-}=$ $\tilde{F}_{j}^{-} \cap F$ for $j=1, \ldots, \nu^{-}$. Applying Lemmas 3.2, 3.3, and 1.2 we get:

$$
\begin{aligned}
c(F) & \leq c\left(F^{+}\right)+c\left(F^{-}\right)+\sum_{j=1}^{\nu^{+}} c\left(F_{j}^{+}\right)+\sum_{j=1}^{\nu^{-}} c\left(F_{j}^{-}\right) \\
& \leq \frac{2 o p t_{k}}{k-l}\left(\frac{2 n}{\beta}+\beta\right) \\
& \leq \frac{4 \sqrt{2 n}}{k-\ell} \text { opt }_{k}
\end{aligned}
$$

Suppose now that the input graph $\mathcal{G}$ contains a $k_{0}$-connected spanning subgraph of cost zero. We can repeatedly apply the above algorithm starting with $\ell=k_{0}$ until $\ell=k-1$, to compute a $k$-connected spanning subgraph of $\mathcal{G}$; the overall cost of the subgraph found will be at most $4 \sqrt{2 n} H\left(k-k_{0}\right) o p t_{k}=$ $O(\sqrt{n} \ln k)$ opt $_{k}$.

For undirected graphs, a $8 \sqrt{2 n} H\left(k-k_{0}\right)$-approximation algorithm easily follows using the reduction due to Khuller and Raghavachari [14] described at the end of Section 1.1.

To finish the proof of Theorem 3.1, let us discuss the implementation and the time complexity of the algorithm. Procedure 1 in our algorithm is similar to the one used in [24], and we can adapt the implementation of [24] as well. We omit the details, but note that for implementing all Procedures 1 in the algorithm, as well as finding minimal edge sets $F \subseteq \tilde{F}$ such that $G+F$ is $(\ell+1)$-connected, $\ell=0, \ldots, k-1$, can be done in $O\left(k^{3} m n^{2}\right)$ total time, see Section 5 in [24]. Using the algorithm of [8], the overall time required for Procedure 2 implementations is $O\left(k^{3} m n^{2} \sqrt{n}\right)$. Note however, that Procedure 2 requires finding a minimumcost edge set to increase the outconnectivity from $\ell$ to $\ell+1$. In the next section we will discuss a primal dual algorithm for this restricted problem, and show that it can be implemented in $O\left(\mathrm{~m}^{2}\right)$ time, see Theorem 4.1 to follow. Thus the total time required for Procedure 2 executions is $O\left(k \sqrt{n} m^{2}\right)$.

\section{ALGORITHM FOR INCREASING OUT- CONNECTIVITY BY 1}

THEOREM 4.1. There exists an algorithm with time complexity $O\left(\mathrm{~m}^{2}\right)$ for the problem of finding a minimum cost augmenting edge set to increase the outconnectivity from $r$ of a directed graph by 1 .

It would be more convenient to consider an equivalent problem of increasing the inconnectivity (recall that a directed graph is $\ell$-inconnected to $r$ if it contains $\ell$ internally disjoint paths from any its node to $r$ ).

Let $G=(V, E)$ be a directed graph which is $\ell$-inconnected to $r$ and let $I$ be an edge set on $V$ disjoint to $E$ (henceforth "graph" stands for directed graph). For $S \subseteq V-r$ let $\bar{S}=\bar{S}_{G}=S^{*}+r$. For $F \subseteq I$ we say that $S \subseteq V-r$ is violated in $G+F$ if $\left|\delta_{E}(S, \bar{r})\right|+n-(|S|+|\bar{S}|)=\ell$ and $\delta_{F}(S, \bar{S})=\emptyset$. Let $\mathcal{S}$ denote the collection of all violated sets in $G$. Consider the following linear program $(\mathrm{P})$ and its dual program (D):

The primal programm:

$$
\begin{array}{lll}
\min & \sum_{e \in I} c_{e} x_{e} & \\
\text { s.t. } & \sum_{\substack{e \in \delta_{I}(S, \bar{S})\\
}} x_{e} \geq 1 \quad \forall S \in \mathcal{S} \\
& x_{e} \geq 0 & \forall e \in I
\end{array}
$$

And the dual programm:

$$
\begin{array}{ll}
\max & \sum_{S \in \mathcal{S}} y_{S} \\
\text { s.t. } & \sum_{S \in \mathcal{S}: e \in \delta_{I}(S, \bar{S})} y_{S} \leq c_{e} \quad \forall e \in I \\
& y_{S} \geq 0 \quad \forall S \in \mathcal{S} .
\end{array}
$$

For $F \subseteq I$, let $x^{F}$ be the characteristic vector of $F$, that is $x_{e}^{F}=1$ if $e \in F$ and $x_{e}^{F}=0$ if $e \in I-F$. By Menger's Theorem, $G+F$ is $(\ell+1)$-inconnected to $r$ if and only if $G+F$ has no violated sets, that is if and only if $\left|\delta_{F}(S, \bar{S})\right| \geq 1$ for every $S \in \mathcal{S}$. Thus $G+F$ is $(\ell+1)$-inconnected to $r$ if and only if $x^{F}$ is a feasible solution to (P).

Given a feasible solution $y$ to (D), we say that an edge $e \in I$ is tight if the corresponding constraint in (D) holds with equality. Our algorithm applies a standard primaldual method using the linear program above. It produces an edge set $F \subseteq I$ such that $x^{F}$ is a feasible solution to $(\mathrm{P})$, and a feasible solution $y$ to $(\mathrm{D})$, such that $x^{F}$ and $y$ satisfy the complementary slackness conditions. Note that the complementary slackness conditions are:

primal conditions: $e \in F \Longrightarrow e$ is tight;

dual conditions: $\quad y_{S}>0 \Longrightarrow\left|\delta_{F}(S, \bar{S})\right|=1$.

The following lemma is well known (the proof is omitted), e.g., see Lemma 6 in [2], where it was proved for undirected graphs.

LEMMA 4.2. The intersection and union of two violated sets are both violated; thus the (inclusion) minimal violated sets are disjoint.

Here is the description of the algorithm. The algorithm has two phases. 
Phase 1 starts with $F=\emptyset$, and applies a sequence of iterations. At each iteration, an edge is selected to be added to $F$, until $G+F$ has no violated sets, that is $G+F$ is $(\ell+1)$-inconnected to $r$. Selecting an edge to add is done as follows. The algorithm maintains (implicitly) a feasible solution $y$ for (D). Initially, $y_{S}=0$ for all $S \in \mathcal{S}$. Now, if $x^{F}$ is still not a feasible solution to (P) (so $G+F$ has at least one violated set), we increase uniformly (possibly by zero) the dual variables $y_{C}$ corresponding to inclusion minimal violated sets in $G+F$ until some edge $e \in I-F$ becomes tight; then $e$ is added to $F$ at this iteration.

Phase 2 applies on $F$ "reverse delete", which means the following. Let $F=\left\{e_{1}, \ldots, e_{j}\right\}$, where $e_{i}$ was added at iteration $i$. For $i=j$ downto 1 , we delete $e_{i}$ from $F$ if $(G+F)-e_{i}$ is $(\ell+1)$-inconnected to $r$. At the end of the algorithm, $F$ is output.

Lemma 4.3. At the end of the algorithm, $x^{F}$ is a feasible solution to $(P), y$ is a feasible solution to $(D)$, and $x^{F}$ and $y$ satisfy the complementary slackness conditions; hence both $x^{F}$ and $y$ are optimal solutions.

Proof. Since $G+F$ is $(\ell+1)$-connected, $x^{F}$ is a feasible solution to $(\mathrm{P})$. It is easy to see that during the algorithm $y$ remains a feasible solution to $(D)$. Since only tight edges enter $F$, after Phase 1 the primal complementary slackness conditions hold for $F$. So, the only part that requires proof is that after Phase 2 the dual complementary slackness conditions hold for $x^{F}$ and $y$.

Consider an arbitrary $S \subseteq V$ with $y_{S}>0$ and an edge $e \in \delta_{F}(S, \bar{S})$.

Claim: There exists a violated set $S_{e}$ in $(G+F)-e$ such that $S \subseteq S_{e}$ and $\delta_{F}\left(S_{e}, \bar{S}_{e}\right)=\{e\}$.

Proof: Such set $S_{e}$ can be chosen as an arbitrary violated set of the graph obtained by deleting (instead of keeping) $e$ at the reverse delete step when $e$ was considered for deletion; note that the algorithm decided to keep $e$, hence this graph is not $(\ell+1)$-inconnected to $r$ and such $S_{e}$ exists. Moreover, since the edges were deleted in the reverse order, $S_{e}$ is violated in $(G+F)-e$. Obviously, $\delta_{F}\left(S_{e}, \bar{S}_{e}\right)=\{e\}$ and $S$ and $S_{e}$ intersect. Finally, to see that $S \subseteq S_{e}$ note that: (i) at any iteration before $e$ was added, $S_{e}$ was violated; (ii) since $y_{S}>0$, there was an iteration before $e$ was added at which $S$ was minimal violated. Hence $S \subseteq S_{e}$, by Lemma 4.2. $\quad \square$

We now show that the dual complementary slackness conditions hold for $x^{F}$ and $y$. Assume to the contrary that there is $S \in \mathcal{S}$ with $y_{S}>0$ such that there are $e^{\prime} \neq e^{\prime \prime}$ and $e^{\prime}, e^{\prime \prime} \in \delta_{F}(S, \bar{S})$. Let $S^{\prime}=S_{e^{\prime}}$ and $S^{\prime \prime}=S_{e^{\prime \prime}}$ be as in the Claim above. Then $S \subseteq S^{\prime} \cap S^{\prime \prime}$; in particular $S^{\prime}$ and $S^{\prime \prime}$ intersect, so $S^{\prime} \cup S^{\prime \prime}$ is violated in $G$, by Lemma 4.2. Thus, there is an edge $e \in \delta_{F}\left(S^{\prime} \cup S^{\prime \prime}, \overline{S^{\prime} \cup S^{\prime \prime}}\right)$. This implies that $e \in \delta_{F}\left(S^{\prime}, \bar{S}^{\prime}\right)$ or $e \in \delta_{F}\left(S^{\prime \prime}, \bar{S}^{\prime \prime}\right)$. Therefore, we must have $e=e^{\prime}$ or $e=e^{\prime \prime}$. Since the tail of each one of $e^{\prime}, e^{\prime \prime}$ is in $S^{\prime} \cap S^{\prime \prime}$, the tail of $e$ is in $S^{\prime} \cap S^{\prime \prime}$. The head of $e$ must be in $\overline{S^{\prime} \cup S^{\prime \prime}}$. We conclude that $e \in \delta_{F}\left(S^{\prime}, \bar{S}^{\prime}\right) \cap$ $\delta_{F}\left(S^{\prime \prime}, \bar{S}^{\prime \prime}\right)$, which is a contradiction since $\delta_{F}\left(S^{\prime}, \bar{S}^{\prime}\right)=\left\{e^{\prime}\right\}$, $\delta_{F}\left(S^{\prime \prime}, \bar{S}^{\prime \prime}\right)=\left\{e^{\prime \prime}\right\}$, and $e^{\prime} \neq e^{\prime \prime}$.

To finish the proof of Theorem 4.1, let us show that the algorithm can be implemented to run in $O\left(\mathrm{~m}^{2}\right)$ time, where $m=|E|+|I|$ (note that $n \ell=O(m)$ ). For $v \in V-r$ let $C_{v}$ be the minimal violated set containing $v$, if such exists. Finding the pairs of sets $C_{v}, \bar{C}_{v}$ for every $V \in V$ can be done in $O(m \ell n)=O\left(m^{2}\right)$ time as follows. For every $v \in V$, we calculate (with the Ford-Fulkerson algorithm, the node-capacity version) a flow of value $\ell$ from $v$ to $r$. In the resulting residual network the set of nodes reachable from $v$, if does not contain $r$, is $C_{v}$. It is easy to see that for each set $C_{v}$, the set $\bar{C}_{v}$ can be found in $O(m)$ time.

We now show that each edge selection step at phase 1 can be implemented in $O(m)$ time. For every minimal violated set $C$ in $G+F$ we keep one such residual network between some "representative node" $v \in C$ and $r$. Note that when an edge is selected to be added to $F$, it affects exactly one minimal violated set. For the corresponding residual network, we add all the edges selected so far and recompute the set of nodes reachable from $v$. If this set contains $r$ or some other representative node, then this network is discarded; otherwise, we update the set $C_{v}, \bar{C}_{v}$. Let us discuss the implementation of the edge selection step. By maintaining residual cost $\bar{c}_{e}=c_{e}-\sum\left\{y_{S}: S \in \mathcal{S}, e \in \delta(S, \bar{S})\right\}$ of each edge $e$ we can find the raise of the dual variables and the corresponding tight edge in $O(m)$ time; among the edges which are in $\delta_{I-F}(C, \bar{C})$ for some minimal violated set $C$, we find an edge of the minimal residual cost, and then update the residual costs of all these edges. Thus the total time required for edge selection steps is $O\left(\mathrm{~m}^{2}\right)$.

Finally, Phase 2 can be implemented in $O\left(m^{2}\right)$ total time. For each candidate for deletion $e=u v \in F$ the time required is $O(m)$; we need to check whether there are still $(\ell+1)$ internally disjoint paths from $u$ to $r$. Since we have a flow of value $\ell$ from $u$ to $r$ in the original graph $G$, and thus in $G+F-e$, all we need is to check whether it can be extended to an $(\ell+1)$-flow from $u$ to $r$ in $G+F-e$. Thus the overall time complexity of Phase 2 is also $O\left(m^{2}\right)$.

Acknowledgments. The authors thank Joseph Cheriyan for helpfull comments

\section{REFERENCES}

[1] V. Auletta, Y. Dinitz, Z. Nutov, and D. Parente, A 2-approximation algorithm for finding an optimum 3-vertex connected spanning subgraph, Journal of Algorithms 32, 1999, 21-30.

[2] J. Cheriyan, T. Jordán, and Z. Nutov, On rooted node-connectivity problems, Algorithmica 30 (special issue on APPROX'98), 2001, 353-375.

[3] J. Cheriyan and R. Thurimella, Approximating minimum-size $k$-connected spanning subgraphs via matching, SIAM J. Comput. 30, no. 2, 2000, 528-560.

[4] J. Cheriyan, S. Vempala, and A. Vetta, Approximation algorithms for minimum-cost k-vertex connected subgraphs, in Proc. 34th ACM Symp. on Theory of Computing (STOC 2002), 306-312.

[5] A. Frank, Connectivity augmentation problems in network design, Mathematical Programming, State of the Art, J. R. Birge and K. G. Murty eds., 1994, 34-63.

[6] A. Frank and T. Jordán, Minimal edge-coverings of pairs of sets, J. Comb. Theory B 65, 1995, 73-110.

[7] A. Frank and É. Tardos, An application of submodular flows, Linear Algebra and its Applications 114/115, 1989, 329-348.

[8] H. N. Gabow, A representation for crossing set families with application to submodular flow 
problems, Proc. 4th Annual ACM-SIAM Symp. on Discrete Algorithms (SODA 1993), 202-211.

[9] Handbook of Combinatorics, Vol. 1 Ch. 7, L. Graham, M. Grötschel, and L.Lovász Eds., 1995.

[10] T. Jordán, On the optimal vertex-connectivity augmentation, J. Combinatorial Theory Series B 63, $1995,8-20$.

[11] T. Jordán, On the existence of $(k, \ell)$-critical graphs, Discrete Math. 179, 1-3, 1998, 273-275.

[12] B. Jackson and T. Jordán, A near optimal algorithm for vertex connectivity augmentation, in Proc. ISAAC 2000, LNCS 1969 (2000), 212-325.

[13] G. Kortsarz and Z. Nutov, Approximating node connectivity problems via set covers, Algorithmica 37, 2003, 75-92.

[14] S. Khuller and B. Raghavachari, Improved approximation algorithms for uniform connectivity problems, J. of Algorithms 21, 1996, 434-450.

[15] M. Kriesell, Upper bounds to the number of vertices in a $k$-critically $n$-connected graph, Graphs and Combinatorics 18, 2002, no. 1, 133-146.

[16] W. Mader, Ecken vom Grad $n$ in minimalen n-fach zusammenhängenden Graphen, Archive der Mathematik 23, 1972, 219-224.

[17] W. Mader, Minimal $n$-fach in minimalen n-fach zusammenhängenden Digraphen, J. Combinatorial Theory Series B, 38, 1985, 102-117.
[18] W. Mader, Endlichkeitsätze für $k$-kritische Graphen (German), Math. Ann. 229, 1977, 143-153.

[19] W. Mader, On $k$-con-critically $n$-connected graphs, $J$. Combin. Theory Ser. B 86, 2002, no. 2, 296-314.

[20] W. Mader, High connectivity keeping sets in $n$-connected graphs, to appear in Combinatorica.

[21] W. Mader, Connectivity and edge-connectivity in finite graphs, Surveys in Combinatorics (Proc. Seventh British Combinatorial Conf., Cambridge, 1979), 66-95, London Math. Soc. Lecture Notes Ser., 38, Cambridge Univ. Press, 1979.

[22] W. Mader, On $k$-critically $n$-connected graphs, Progress in Graph Theory (Waterloo, Ont., 1982), 389-398, Academic Press, Toronto, On, 1984.

[23] B. Maurer and P. Slater, On $k$-critical, $n$-connected graphs, Discrete Mathematics 20, 1988, 255-262.

[24] R. Ravi and D. P. Williamson, An approximation algorithm for minimum-cost vertex-connectivity problems, Algorithmica 18, 1997, 21-43.

[25] R. Ravi and D. P. Williamson, Erratum: An approximation algorithm for minimum-cost vertex-connectivity problems, Algorithmica 34(1), 2002, 98-107.

[26] J. Su, Proof of Slater's conjecture on $k$-critical n-connected graphs, Kexue Tongbao (English Ed.) 33, 1988, no. 20, $1675-1678$. 